\title{
Circulating endothelial progenitor cells in metronomic chemotherapy using irinotecan and/or bevacizumab for colon carcinoma: Study of their clinical significance
}

\author{
HIDETSUGU MURAKAMI ${ }^{1}$, YUTAKA OGATA ${ }^{1,2}$, YOSHITO AKAGI ${ }^{1}$, \\ NOBUYA ISHIBASHI ${ }^{1}$ and KAZUO SHIROUZU ${ }^{1}$ \\ ${ }^{1}$ Department of Surgery, Kurume University School of Medicine; \\ ${ }^{2}$ Department of Surgery, Kurume University Medical Center, Kurume, Japan
}

Received January 14, 2011; Accepted March 23, 2011

DOI: $10.3892 /$ etm.2011.253

\begin{abstract}
The aim of the present study was to clarify the antitumor efficacy of metronomic chemotherapy using irinotecan (CPT-11) combined with or without bevacizumab against colon cancer, and the significance of circulating endothelial cell (CECs) and endothelial progenitor cells (CEPs) as a surrogate marker for metronomic chemotherapy. KM12SM cells were implanted into the subcutis of nude mouse. After confirming that the implanted tumors had grown $5 \mathrm{~mm}$ in size, group A received an intraperitoneal injection of $40 \mathrm{mg} /$ kg CPT-11 every two weeks for 4 weeks [conventional maximum-tolerated dose (MTD)], group B received $10 \mathrm{mg}$ / $\mathrm{kg}$ twice weekly (metronomic), group $\mathrm{C}$ received $10 \mathrm{mg} / \mathrm{kg}$ twice weekly combined with $5 \mathrm{mg} / \mathrm{kg}$ bevacizumab twice weekly (metronomic + anti-angiogenic), and the control group received $0.2 \mathrm{ml}$ of PBS every week. Serial changes of CECs and CEPs in peripheral blood and microvessel density (MVD) in the tumor tissues were evaluated. The results showed that the antitumor activity in group B and in group C was significantly higher than that in group A. A significant inhibition in CEPs on day 15 in the metronomic therapy groups B and $\mathrm{C}$ was noted when compared to that in the control group, while there was no significant difference in CECs and CEPs between the groups on days 4 and 8 . The MVD on day 15 in metronomic groups was significantly lower than that in group A. In conclusion, metronomic chemotherapy of CPT-11 with or without bevacizumab for colon cancer was more effective than the MTD therapy via anti-angiogenic effects. Sequential measurement of CEPs may be a predictive factor for the efficacy and a decisive factor for the optimal dose of metronomic therapy in colon cancer.
\end{abstract}

Correspondence to: Professor Yutaka Ogata, Department of Surgery, Kurume University Medical Center, 155-1 Kokubu-machi, Kurume 839-0863, Japan

E-mail: yogata@med.kurume-u.ac.jp

Key words: circulating endothelial cell, irinotecan, anti-angiogenesis, maximum-tolerated dose, surrogate marker for angiogenesis

\section{Introduction}

Angiogenesis plays a pivotal role in tumorigenesis and metastasis (1). Tumor angiogenesis is a complex process and is based on the concept that a tumor requires a vascular blood supply to grow beyond 1 or $2 \mathrm{~mm}(2,3)$. Tumors that do not establish a neovascular supply may remain dormant for a long time (4). Neovascularization has been thought to result exclusively through proliferation, migration and remodeling of fully differentiated endothelial cells derived from pre-existing blood vessels. In addition, vascular endothelial growth factor (VEGF) has been found to induce mobilization of bone marrow-derived endothelial progenitor cells resulting in increased numbers of differentiated endothelial progenitor cells and augmented neovascularization $(5,6)$.

Conventional cytotoxic chemotherapeutic drugs are sensitive to endothelial cells in addition to directly sacrificing or inhibiting the proliferation of rapidly dividing tumor cells (7). However, conventional chemotherapy, which is administered at the more toxic maximum-tolerated dosage (MTD), requires 2- to 3-week rest periods between successive cycles of therapy. The anti-angiogenic efficacy of chemotherapy appears to be optimized by administering comparatively low dosages of the drug on a frequent (daily, several times a week or weekly) or continuous schedule, with no extended interruptions. This concept is sometimes referred to as 'metronomic' chemotherapy (8). In such a situation, mature circulating endothelial cells (CECs) and endothelial progenitor cells (CEPs) have been used as a potentially useful surrogate marker for antiangiogenic activity (9).

Recently, various drugs have been shown to have significant anti-angiogenic activity when administered at a low dosage using a metronomic schedule $(10,11)$. Irinotecan (CPT11), which has resulted in improved prognosis of patients with metastatic colorectal cancer $(12,13)$, is always administered using a therapeutic MTD approach; thus, the antitumor and anti-angiogenic efficacy of metronomic CPT-11 administration is unknown.

Humanized monoclonal antibody bevacizumab against VEGF demonstrated an antitumor effect through its administration combined with chemotherapy using CPT-11 and 
Table I. Administration schedules of CPT-11 and bevacizumab.

\begin{tabular}{lcccc}
\hline Treatment groups & $\begin{array}{c}\text { Dose of CPT-11 } \\
(\mathrm{mg} / \mathrm{kg})\end{array}$ & $\begin{array}{c}\text { Dose of bevacizumab } \\
(\mathrm{mg} / \mathrm{kg})\end{array}$ & Application & $\begin{array}{r}\text { Total dose of CPT-11 } \\
\text { over 4 weeks }(\mathrm{mg} / \mathrm{kg})\end{array}$ \\
\hline Group A (Conv-40) & 40 & & Day 1, 15 i.p. & 80 \\
Group B (Metro-10) & 10 & 5 & Twice weekly i.p. & 80 \\
Group C (Metro-10 + Beva) & 10 & Twice weekly i.p. & 80
\end{tabular}

Conv, conventional; Metro, metronomic; Beva, bevacizumab; i.p., intraperitoneal.

5-FU/LV in a phase III trial for advanced colorectal cancer $(14,15)$. Angiogenesis inhibitors also have effects on CECs and CEPs, and these changes have emerged as a potentially useful surrogate marker (16). However, the serial change in the number of CECs/CEPs in chemotherapy, in particular in metronomic chemotherapy is still unknown. In the present study, we investigated the serial change of CECs/CEPs, and the relationship between antitumor efficacy and CECs/CEPs, in metronomic chemotherapy using CPT-11 combined with or without bevacizumab for colon cancer.

\section{Materials and methods}

Drugs. Bevacizumab was a kind gift from Genentech (South San Francisco, CA). CPT-11 was a gift from Yakult Honsha (Tokyo, Japan). CPT-11 solution was freshly prepared in $0.9 \%$ saline at a concentration of $1 \mathrm{mg} / \mathrm{ml}$.

Cell culture. The human colon carcinoma cell line KM12SM, which produces a high level of VEGF in monolayer culture (supernatant: $2822 \mathrm{pg} / \mathrm{ml} / 10^{6} / 48 \mathrm{~h}$, unpublished data), was kindly provided by Dr M. Nakajima (Johnson \& Johnson KK, Tokyo, Japan). The tumor cells were harvested from subconfluent cultures by a 5-min treatment with trypsin-EDTA (Invitrogen, Tokyo, Japan). The dislodged cells were first washed in RPMI-1640 (Invitrogen) supplemented with 10\% fetal bovine serum and re-suspended in phosphate-buffered saline (PBS) for injection. Only single cells in suspension with $>90 \%$ viability were used for the injections.

Animals. Male BALB/c/nu/nu mice, aged 4 weeks, were purchased from Clea Japan, Inc. (Tokyo, Japan). The mice were maintained in a laminar-flow cabinet under specific pathogen-free conditions and were used for experiments at the age of 5 weeks. The mice were maintained in facilities according to the regulations and standards of the Kurume University School of Medicine.

Tumor xenografts and assessment of antitumor effects. A total of $1 \times 10^{6} \mathrm{KM} 12 \mathrm{SM}$ cells/PBS was transplanted into the subcutis of the dorsal skin in each nude mouse. The maximum tumor diameter was set at $5 \mathrm{~mm}$ and then CPT-11 combined with or without bevacizumab was administered intraperitoneally at a dosage of $10-40 \mathrm{mg} / \mathrm{kg}$ of CPT-11 [up to one-fourth and one-sixteenth the dosage of the $\mathrm{LD}_{50}$ of $\left.177.5 \mathrm{mg} / \mathrm{kg}(17)\right]$ and $5 \mathrm{mg} / \mathrm{kg}$ of bevacizumab for 28 days. After confirming that the implanted tumor had grown $5 \mathrm{~mm}$ in size, mice were divided into 4 groups. Group A received $40 \mathrm{mg} / \mathrm{kg}$ of CPT-11 every two weeks (Conv-40), and group B received $10 \mathrm{mg} / \mathrm{kg}$ of CPT-11 twice weekly (Metro-10). Group C received $10 \mathrm{mg} /$ $\mathrm{kg}$ of CPT-11 twice weekly combined with $5 \mathrm{mg} / \mathrm{kg}$ of bevacizumab twice weekly (Metro-10 + Beva). The control group received $0.2 \mathrm{ml}$ of PBS every week (Table I). We calculated the body weight of each mouse from day 0 to 29 , and these data were used as an indicator of side effects. The tumor size was measured twice weekly using calipers, and the tumor volume was calculated by the formula: [(Maximum tumor diameter $^{2}$ x Minimum tumor diameter/2]. We then resected the tumors 29 days after the start of the drug administration, and the tumors were fixed with $10 \%$ formalin for histological examination.

Measurement of CECs and CEPs by flow cytometry. Mice were euthanized with diethyl ether on days $0,4,8$ and 15 , in each group, and heparinized blood was obtained from the heart for CEC and CEP evaluation. CECs and CEPs were counted using a FACSVantage SE flow cytometer (BD Biosciences, San Jose, CA), and the acquired data were analyzed with FlowJo version 6.3.2 flow cytometry analysis software (Tree Star, Inc., Ashland, OR). Heparinized whole blood was hemolyzed and stained with anti-mouse CD45 monoclonal antibody, anti-mouse Flk-1 antibody, anti-mouse CD31 monoclonal antibody and anti-mouse CD117 monoclonal antibody (all from BD Bioscience, San Diego, CA). After red cell lysis, cell suspensions were evaluated by a FACSVantage SE using analysis gates designed to exclude dead cells, platelets and debris. $\mathrm{CD} 45^{+}$cells were excluded by gating, and then $\mathrm{CD} 31^{+}$ and Flk $-1^{+}$cells were separated from the CD45- cells. Among these cells, CD117 ${ }^{-}$cells were regarded as CECs, and CD117 ${ }^{+}$ cells were regarded as CEPs (Fig. 1). After acquisition of at least 100,000 cells/sample, analyses were considered as informative when adequate numbers of events (i.e., $>50$, typically 100-200) were collected in the CEC and CEP enumeration gates $(18,19)$.

Immunohistochemistry for microvessels and assessment of microvessel density. The dorsal subcutaneous tumor was fixed by formalin and embedded into paraffin. Serial sections $3 \mu \mathrm{m}$ were cut from each block. One section was stained using hematoxylin and eosin (H\&E), and a second was immunostained for CD31. Immunoreactivities were determined using the avidin-biotin peroxidase complex method (Vector Laboratories, Burlingame, CA) using anti-mouse CD31 (Abcam, Cambridge, MA) at no dilution as the primary anti- 
A

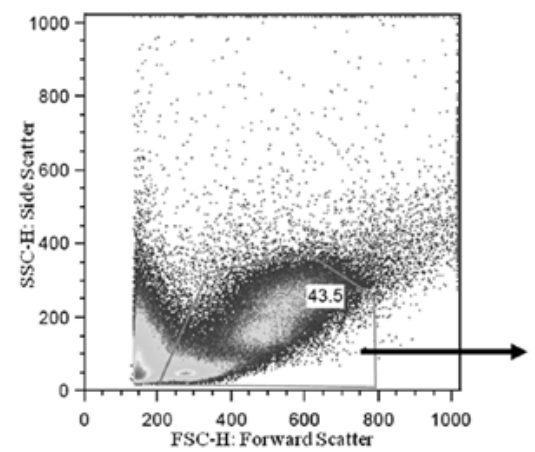

C

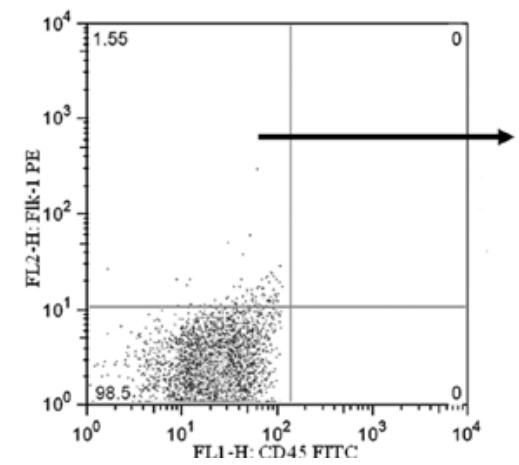

B

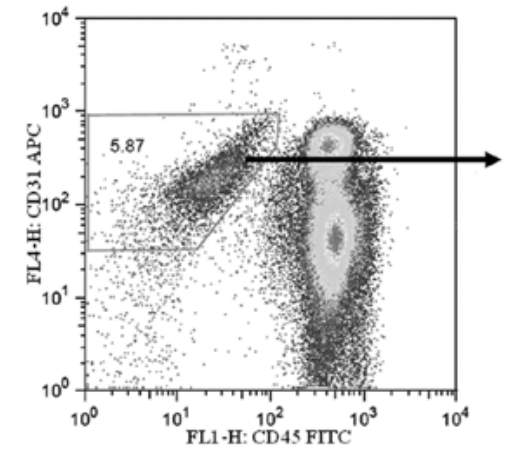

D

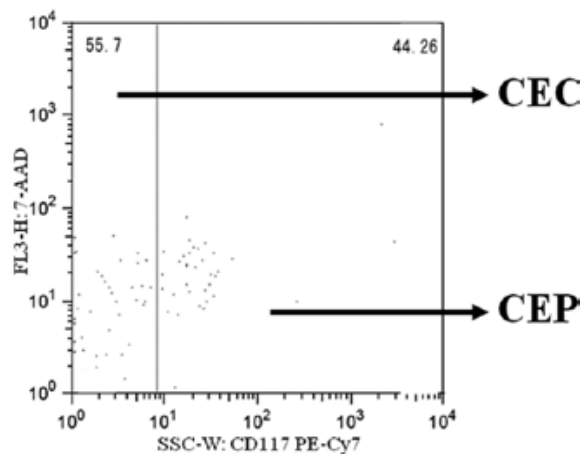

Figure 1. Representative flow cytometric evaluation of CEC and CEP enumeration. (A) Initial gate used to exclude red cells, platelets and debris. (B) Selection of CD45 and CD31 $1^{+}$cells. (C) Gate for the enumeration of CD45, CD31 and Flk-1+ cells. (D) Gate for enumeration of the CD45, CD31 ${ }^{+}, \mathrm{Flk}^{+} 1^{+}$and $\mathrm{CD} 117^{-}$ cells (CECs) and CD45, CD31 $1^{+}$, Flk- $1^{+}$and $\mathrm{CD} 117^{+}$cells (CEPs).

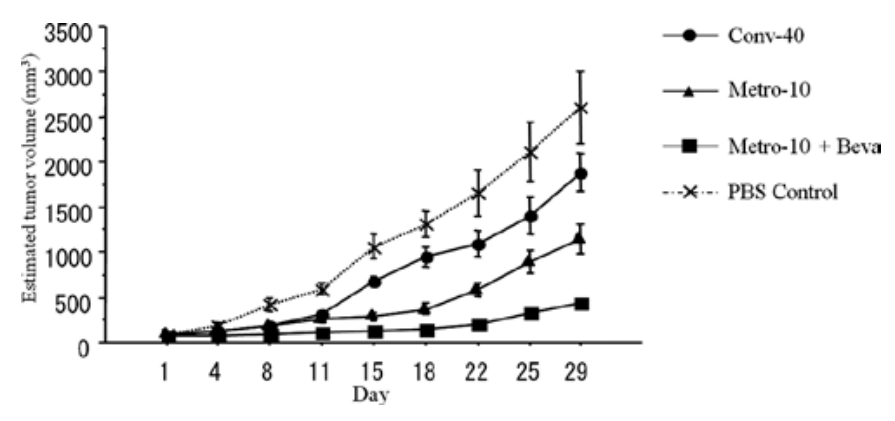

Figure 2. Growth curves of the subcutaneous (KM12SM cell) tumors implanted in nude mouse. The maximum tumor diameter was set at $5 \mathrm{~mm}$, and CPT-11 combined with or without bevacizumab was administered intraperitoneally.

body. Hematoxylin was used as the counterstain. The negative controls used reagents except for the primary antibody. Positive staining of a small tubular formation for CD31 was defined as a macrovessel, and the microvessel density (MVD) was assessed as the average number of vessels $/ \mathrm{mm}^{2}$, over three areas, at x200 magnifi-cation (20).

Statistical analysis. The deta were analyzed using the Student's t-test. The tumor volume was analyzed using two-way repeated ANOVA. A P-value $<0.05$ was considered statistically significant. Analyses were computed using the StatView v.5.0 software (SAS Institute Inc., Cary, NC).

\section{Results}

Growth inhibition of the tumors implanted into the mouse subcutis. Conventional treatment of CPT-11 (Conv-40) showed significantly higher antitumor activity compared with the PBS

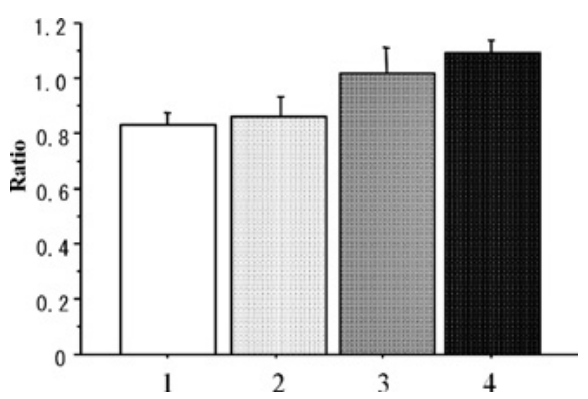

Figure 3. The weight-loss ratio (day 29/day 0): 1, PBS control; 2, Conv-40 group; 3, Metro-10 group; 4, Metro-10 + Beva group.

control group $(\mathrm{P}=0.019)$. In addition, metronomic treatment using CPT-11 (Metro-10) showed more effective antitumor activity compared to the conventional (Conv-40) group $(\mathrm{P}<0.01)$. An additive antitumor effect was found when bevacizumab was combined with metronomic chemotherapy using CPT-11 (Metro-10 + Beva) ( $\mathrm{n}=10$ in each group) $(\mathrm{P}<0.01)$ (Fig. 2).

The weight-loss ratio (day 29/day 0) was statistically lower in the conventional group (Conv-40) than that in the metronomic-treated (Metro-10 \pm Beva) groups $(\mathrm{P}=0.004)$, although there was no significant difference in the weight loss ratio between the conventional (Conv-40) group and that in the PBS control group ( $\mathrm{n}=7$ in each group) $(\mathrm{P}=0.909)$ (Fig. 3).

Serial changes of CECs and CEPs. CEC and CEP enumeration by flow cytometry is depicted in Fig. 1. The numbers of CECs in the control group and the conventional (Conv-40) group on day 4 and 8 showed no difference compared to the numbers on day 0 , while the numbers of CECs on day 4 and 8 tended to 

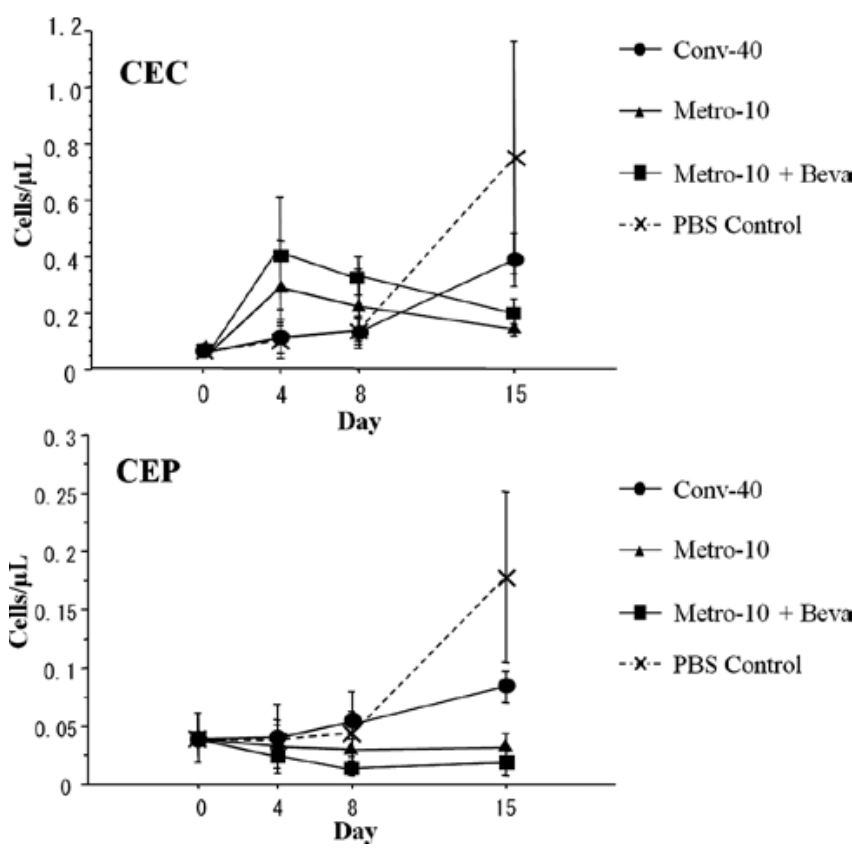

Figure 4. CEC and CEP kinetics before and after treatment in BALB/c/nu/nu mice implanted with KM12SM cell tumors.
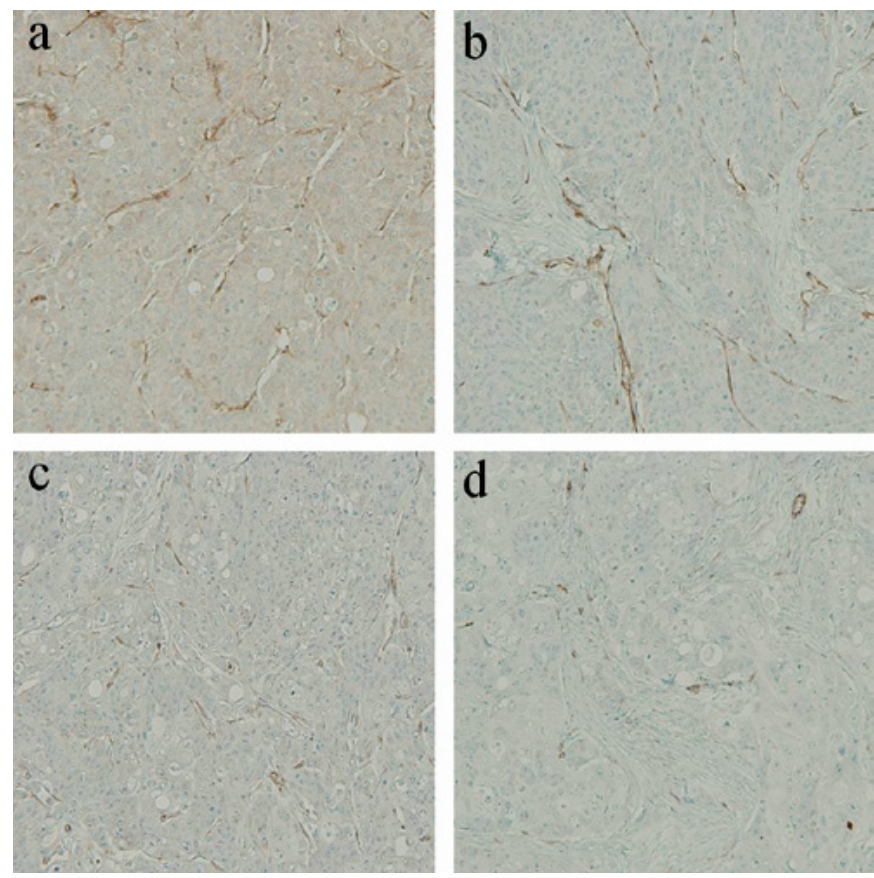

Figure 5. Immunohistochemical staining for CD31 of subcutaneously implanted tumors: (a) PBS control, (b) Conv-40, (c) Metro-10, (d) Metro-10 + Beva.

increase in the metronomic therapy (Metro-10 \pm Beva) groups. While the numbers of CECs increased in the control group and the conventional (Conv-40) group on day 15, the numbers of CECs on day 15 in the metronomic therapy groups tended to decrease compared to the numbers on day 4 and 8 , but did not reach significance.

There was no significant difference in the number of CEPs between each group on day 4 and 8 . However, the numbers of CEPs tended to decrease in the metronomic therapy groups on day 8 compared to those on day 0 . Although a statistically

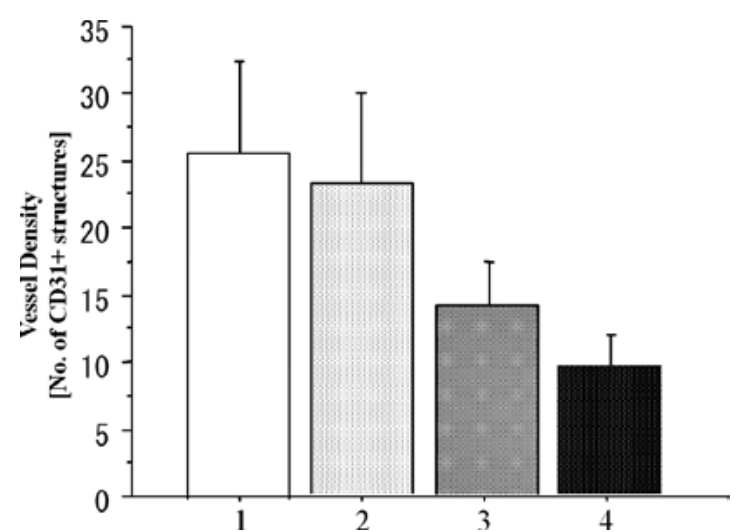

Figure 6. Quantification of the microvessel density in the implanted KM12SM cell tumors on day 15: 1, PBS control; 2, Conv-40; 3, Metro-10; 4, Metro-10 + Beva.

significant increase in the numbers of CEPs in the control and conventional (Conv-40) group on day 15 was noted compared to those on day 0,4 and $8(\mathrm{P}=0.028)$, there was no increase in numbers of CEPs in the metronomic therapy groups $(n=5$ in each group) even on day 15 (Fig. 4).

Analysis of MVD. To investigate the antitumor mechanism of the metronomic CPT-11 treatment combined with or without bevacizumab, we evaluated the MVD in the implanted colon cancer tumors in the subcutis on day 15 after the beginning of drug administration (Fig. 5). The MVD values on day 15 in the metronomic treatment (Metro-10 \pm Beva) groups were significantly lower than that in the conventional (Conv-40) group $(\mathrm{P}<0.001)$, although there was no significant difference between the conventional group and the PBS control group $(\mathrm{P}=0.173)$. Additive effects of the inhibition of vascularization were found when bevacizumab was combined with metronomic treatment of CPT-11 (Metro-10 + Beva vs. the Metro-10; $\mathrm{P}<0.001)(\mathrm{n}=7$ in each group) (Fig. 6).

\section{Discussion}

The purpose of the present study was to clarify the efficacy of metronomic chemotherapy using CPT-11 and its combination with bevacizumab, a specific anti-angiogenic agent, and the significance of CECs and CEPs as a surrogate marker for efficacy in metronomic chemotherapy/anti-angiogenic therapy for colon cancer. The concept of metronomic chemotherapy was summarized by Kerbel and Kamen (8) and Klement et al (21) as follows. (i) Conventional cytotoxic anticancer drugs have anti-angiogenic effects which could contribute to their efficacy. (ii) The anti-angiogenic effects of chemotherapy appear to be optimized by administering such drugs 'metronomically', in other words in small dosages using a frequent schedule (daily, several times a week or weekly) in an uninterrupted manner, over a relatively long period. (iii) Conventional chemotherapy, which is administered at a more toxic MTD, requires 2- to 3 -week rest periods between successive cycles of therapy (which counteracts the potential for sustained therapeutically effective anti-angiogenic effects). (iv) In preclinical models, metronomic chemotherapy can be effective in treating tumors in which cancer cells have developed resistance to the same chemo- 
therapeutics in an MTD administration (which also has the advantage of being less acutely toxic, therefore making a more extended treatment possible). (v) The efficacy of metronomic chemotherapy can be significantly increased when administered in combination with anti-angiogenic drugs, such as antibodies against VEGF or VEGF receptor 2. Finally, (vi) some metronomic chemotherapy regimens induce sustained suppression in CEPs and increase the levels of the endogenous angiogenesis inhibitor thrombospondin-1, both of which can suppress neovascularization.

In our experiment, the metronomic dispensing method of CPT-11 showed a higher tumor proliferation-controlling effect associated with reduced tumor MVD in nude mice transplanted with KM12SM colon carcinoma cells when compared with the conventional dispensing method. Moreover, the tumor proliferation-controlling effect of metronomic administration of CPT-11 was significantly increased when combined with bevacizumab, an anti-angiogenic agent. In addition, in the metronomic administration groups weight loss as an adverse effect was milder compared with that in the conventional MTD administration group. These results from our colon cancer model also support the concept of low-dosage metronomic chemotherapy suggesting the ability of long-term administration and tumor proliferation control.

It has been reported that, although the numbers of CEPs markedly increase during rest periods between MTD administrations of a chemotherapeutic agent to tumor-bearing mice, CEPs are absent during the metronomic administration and the development of tumors was not noted (18). We also investigated the serial changes in CECs/CEPs and their relationship with tumor vasculature (MVD) after treatment with CPT-11 and the vascular-targeting agent bevacizumab. Our data provide evidence that metronomic administration of CPT-11 and its combination with bevacizumab can have opposing effects in the early phase on days 4 and 8 and then similar effects in the late phase on day 15 on the number of CEPs and mature CECs just prior to the next MTD administration. Namely, the metronomic chemotherapy tended to increase the numbers of mature CECs and to decrease the numbers of CEPs in the early phase after the beginning of treatment (day 4 and 8), and tended to decrease both CECs and CEPs in the late phase after the beginning of treatment (day 15) in the KM12SM cell tumor-bearing mice. In particular, the difference in the numbers of CEPs in the late phase between the metronomic chemotherapy and conventional MTD chemotherapy was statistical significant. The small numbers of CEPs was associated with a concomitant inhibition in tumor vasculature and in tumor growth, suggesting that continuous suppression of CEPs may be a marker for anti-angiogenic activity, including metronomic chemotherapy in a clinical situation.

Our results support the conclusion that the antitumor effects of low dosage metronomic chemotherapy are attributable, at least in part, to a mechanism involving inhibition of tumor blood vessel formation. In addition to anti-angiogenic mechanisms in which fully differentiated endothelial cells are growth-inhibited and/or sacrificed by metronomic low-dosage chemotherapy (6), an anti-vasculogenic process may also be involved which is mediated by reduced CEP mobilization and viability. It is also interesting to consider whether MTD chemotherapy may sometimes accelerate tumor (re)growth and drug resistance by increased mobilization of CEPs. This may also help explain the robust reversal of the damage inflicted by MTD chemotherapy on tumor blood vessel endothelial cells as noted by Browder et al (22). An influx of mobilized CEPs during the rest periods between cycles of MTD therapy may replace damaged or sacrificed endothelial cells. In this regard, evaluating the mobilization, viability, and levels of CEPs detected in cancer patients treated with low-dosage metronomic chemotherapy regimens, (e.g., daily low-dosage oral chemotherapy and twice weekly oral methotrexate for breast cancer (23) or leukeran for lymphoma) (24) may be of considerable interest. Such studies and our data may provide a surrogate marker with which to monitor the anti-vasculogenic effects of metronomic chemotherapy protocols. In murine studies, the anti-angiogenic agent endostatin decreased the number of viable CEPs (25), whereas cyclophosphamide either induced or inhibited CEPs depending on whether it was administered in a conventional (every 21 days) or metronomic (every 6 days) dosing schedule (18).

With regard to the increase in the number of CECs early after the start of metronomic chemotherapy, it was found that mature CECs increased after 3 days of treatment with ZD6474 targeting the tumor vasculature in tumor-bearing mice but not in non-tumor-bearing mice (16), suggesting that the increase in mature CECs was due, at least in part, to the presence of the tumor and that ZD6474 or metronomic chemotherapy has at least some degree of selectivity for tumor endothelial cells rather than endothelial cells from normal vasculature. On the other hand, metronomic chemotherapy or anti-angiogenic therapy decreased the number of CECs on day 15 as well as the number of CEPs, while the CECs increased on day 15 in the control group and the MTD conventional chemotherapy group. The changes in number of CECs were similar to the changes of CEPs on day 15 after each treatment and in the control. These data suggest that mature CECs may originate from differentiation of CEPs in addition to the sloughing of tumor endothelial cells. Thus metronomic chemotherapy can consistently inhibit an increase in CEPs for a long time, while the number of CECs may be dependent on various factors such as anti-angiogenic efficacy, tumor volume, the status of tumor vasculature and time after chemotherapy, resulting in large individual variations in the number of CECs.

Recently, oral daily fluoropyrimidines such as capecitabine and UFT/LV have not been proven inferior to bolus and/ or infusion MTD chemotherapy using 5-FU in randomized control studies for colon cancer $(26,27)$. Also combination therapies of oral fluoropyrimidine and oxaliplatin/CPT-11 have been developed for colorectal cancer $(28,29)$. Oral fluoropyrimidine would be a typical agent for metronomic chemotherapy (30). We previously reported the safety and efficacy of metronomic chemotherapy using low-dosage weekly CPT-11 and daily 5'-deoxy-5-fluorouridine, an intermediate metabolite of capecitabine, for advanced colorectal cancer (31). However, one of the major problems is a definition of the optimal dosage based on the concept of metronomic chemotherapy. This is a key reason why metronomic chemotherapy has not been widely adopted in clinical trials. Our data suggests that one possible means of determining the recommended dosage for metronomic chemotherapy is to monitor the serial change of CEPs rather than that of unstable 
CECs. The optimal dosage for metronomic chemotherapy can be established as the lowest level which is associated with no increase or decrease in the number of CEPs for an individual patient.

We conclude that metronomic chemotherapy using CPT-11 against colon cancer was more effective than conventional therapy, via an anti-angiogenic effect. The combination with the specific anti-angiogenic agent, bevacizumab, may realize the advantage of metronomic chemotherapy. Measurement of CEPs may be a consistent predictive factor for metronomic chemotherapy in colon cancer. The assessment of serial changes in CEP values is recommended in clinical trials of metronomic chemotherapy.

\section{Acknowledgements}

This study was supported by a Grant-in-Aid for Scientific Research (C) (no. 20591597) from the Ministry of Education, Culture, Sports, Science and Technology, of Japan.

\section{References}

1. Eskens FA: Angiogenesis inhibitors in clinical development; where are we now and where are we going? Br J Cancer 90: 1-7, 2004.

2. Folkman J: Tumor angiogenesis: therapeutic implications. N Engl J Med 285: 1182-1186, 1971.

3. Warren RS, Yuan H, Matli MR, et al: Regulation by vascular endothelial growth factor of human colon cancer tumorigenesis in a mouse model of experimental liver metastasis. J Clin Invest 95: 1789-1797, 1995

4. Takahashi Y, Ellis LM and Mai M: The angiogenic switch of human colon cancer occurs simultaneous to initiation of invasion. Oncol Rep 10: 9-13, 2003.

5. Asahara T, Murohara T, Sullivan A, et al: Isolation of putative progenitor endothelial cells for angiogenesis. Science 275: 964-967, 1997.

6. Asahara T, Takahashi T, Masuda H, et al: VEGF contributes to postnatal neovascularization by mobilizing bone marrowderived endothelial progenitor cells. EMBO J 18: 3964-3972, 1999.

7. Bocci G, Francia G, Man S, et al: Thrombospondin 1, a mediator of the anti-angiogenic effects of low-dose metronomic chemotherapy. Proc Natl Acad Sci USA 100: 12917-12922, 2003.

8. Kerbel RS and Kamen BA: The anti-angiogenic basis of metronomic chemotherapy. Nat Rev Cancer 4: 423-436, 2004.

9. Monestiroli S, Mancuso P, Burlini A, et al: Kinetics and viability of circulating endothelial cells as surrogate angiogenesis marker in an animal model of human lymphoma. Cancer Res 61: 4341-4344, 2001.

10. Bocci G, Nicolaou KC and Kerbel RS: Protracted low-dose effects on human endothelial cell proliferation and survival in vitro reveal a selective anti-angiogenic window for various chemotherapeutic drugs. Cancer Res 62: 6938-6943, 2002.

11. Shaked Y, Emmenegger U, Man S, et al: Optimal biologic dose of metronomic chemotherapy regimens is associated with maximum anti-angiogenic activity. Blood 106: 3058-3061, 2005.

12. Shimada Y, Yoshino M, Wakui A, et al: Phase II study of CPT-11, a new camptothecin derivative, in metastatic colorectal cancer. CPT-11 Gastrointestinal Cancer Study group. J Clin Oncol 11: 909-913, 1993

13. Rothenberg ML, Cox JV, deVore RF, et al: A multicenter, phase II trial of weekly irinotecan (CPT-11) in patients with previously treated colorectal carcinoma. Cancer 85: 786-795, 1999.
14. Kim KJ, Li B, Winer J, et al: Inhibition of vascular endothelial growth factor-induced angiogenesis suppresses tumor growth in vivo. Nature 362: 841-844, 1993.

15. Gerber HP and Ferrara N: Pharmacology and pharmacodynamics of bevacizumab as monotherapy or in combination with cytotoxic therapy in preclinical studies. Cancer Res 65: 671-680, 2005.

16. Beaudry P, Force J, Naumov G, et al: Differential effects of vascular endothelial growth factor receptor-2 inhibitor ZD6474 on circulating endothelial progenitors and mature circulating endothelial cells: implications for use as a surrogate marker of anti-angiogenic activity. Clin Cancer Res 11: 3514-3522, 2005.

17. Nitta K, Yokokura T, Sawada S, et al: Antitumor activity of novel derivatives of camptothecin. Jpn J Cancer Chemother 14: 850-857, 1987.

18. Bertolini F, Paul S, Mancuso P, et al: Maximum tolerable dose and low-dose metronomic chemotherapy have opposite effects on the mobilization and viability of circulating endothelial progenitor cells. Cancer Res 63: 4342-4346, 2003.

19. Capillo M, Mancuso P, Gobbi A, et al: Continuous infusion of endostatin inhibits differentiation, mobilization, and clonogenic potential of endothelial cell progenitors. Clin Cancer Res 9: 377-382, 2003

20. Mizobe T, Ogata Y, Murakami H, et al: Efficacy of the combined use of bevacizumab and irinotecan as a postoperative adjuvant chemotherapy in colon carcinoma. Oncol Rep 20: 517-523, 2008.

21. Klement G, Baruchel S, Rak J, et al: Continuous low-dose therapy with vinblastine and VEGF receptor-2 antibody induces sustained tumor regression without overt toxicity. J Clin Invest 105: R15-R24, 2000

22. Browder T, Butterfield CE, Kräling BM, et al: Anti-angiogenic scheduling of chemotherapy improves efficacy against experimental drug-resistant cancer. Cancer Res 60: 1878-1886, 2000.

23. Colleoni M, Rocca A, Sandri MT, et al: Low-dose oral methotrexate and cyclophosphamide in metastatic breast cancer: antitumor activity and correlation with vascular endothelial growth factor levels. Ann Oncol 13: 73-80, 2002.

24. De Bont ES, Guikema JE, Scherpen F, et al: Mobilized human $\mathrm{CD} 34^{+}$hematopoietic stem cells enhance tumor growth in a nonobese diabetic/severe combined immunodeficient mouse model of human non-Hodgkin's lymphoma. Cancer Res 61: 7654-7659, 2001.

25. Schuch G, Heymach JV, Nomi M, et al: Endostatin inhibits the vascular endothelial growth factor-induced mobilization of endothelial progenitor cells. Cancer Res 63: 8345-8350, 2003.

26. Twelves C; Xeloda Colorectal Cancer group: Capecitabine as first-line treatment in colorectal cancer. Pooled data from two large, phase III trials. Eur J Cancer 38 (Suppl. 2): S15-S20, 2002.

27. Douillard JY, Hoff PM, Skillings JR, et al: Multicenter phase III study of uracil/tegafur and peroral leucovorin versus fluorouracil and leucovorin in patients with previously untreated metastatic colorectal cancer. J Clin Oncol 20: 3605-3616, 2002.

28. Santini D, Vincenzi B, Schiavon G, et al: Chronomodulated administration of oxaliplatin plus capecitabine (XELOX) as first line chemotherapy in advanced colorectal cancer patients: phase II study. Cancer Chemother Pharmacol 59: 613-620, 2007.

29. Goto A, Yamada Y, Yasui H, et al: Phase II study of combination therapy with S-1 and irinotecan in patients with advanced colorectal cancer. Ann Oncol 6: 968-973, 2006.

30. Kato H, Ichinose Y, Ohta M, et al: A randomized trial of adjuvant chemotherapy with uracil-tegafur for adenocarcinoma of the lung. N Engl J Med 350: 1713-1721, 2004.

31. Ogata Y, Sasatomi T, Mori S, et al: Significance of thymidine phosphorylase in metronomic chemotherapy using CPT-11 and doxifluridine for advanced colorectal carcinoma. Anticancer Res 27: 2605-2612, 2007. 